\title{
Safety, Handling and Electrical Performances of Bradycardia Leads in Acute Conditions: Results from the FINE Registry
}

\author{
Henri Benkemoun ${ }^{*}$, Sébastien Prevot², José Antonio Lapuerta ${ }^{3}$, Xavier Dessenne4, \\ Pierre Khattar ${ }^{5}$, Mara Rolando', Philippe Deutsch ${ }^{7}$, Roger Villuendas ${ }^{8}$ \\ ${ }^{1}$ Saint Pierre Clinic, Perpignan, France \\ ${ }^{2}$ Clairval Clinic, Marseille, France \\ ${ }^{3}$ Cabueñes Hospital, Gijon, Spain \\ ${ }^{4}$ Mousseau Clinic, Evry, France \\ ${ }^{5}$ Public Hospital, Lorient, France \\ ${ }^{6}$ SORIN CRM SAS, Lausanne, Switzerland \\ ${ }^{7}$ Department of Cardiology, Public Hospital, Saint-Malo, France \\ ${ }^{8}$ Germans Trias i Pujol Hospital, Carretera de Canyet, Badalona (Barcelona), Spain \\ Email: "henri.benkemoun@wanadoo.fr
}

Received 30 September 2015; accepted 14 November 2015; published 17 November 2015

Copyright (C) 2015 by authors and Scientific Research Publishing Inc.

This work is licensed under the Creative Commons Attribution International License (CC BY). http://creativecommons.org/licenses/by/4.0/

(c) (i) Open Access

\section{Abstract}

Background: Beflex is an active fixation atrial and ventricular lead with a retractable screw; $\mathrm{X}$-Fine is a passive fixation ventricular lead. These two bradycardia lead models were evaluated in the FINE study, an observational prospective trial conducted in France and Spain. Methods: Patients enlisted for pacemaker or defibrillator implants were enrolled. The primary objective was to assess acute dislodgement rates at the 3-month follow-up visit. Safety and electrical performances of the leads were assessed in acute conditions at implant and at the follow-up visit up to three months later. A handling questionnaire was submitted to implanting investigators immediately after implant. Results: A total of 2254 patients were enrolled in 95 centers; investigators implanted 1153 active atrial leads, mainly in the right atrium; 1021 active right ventricular leads, mainly in the septum and $\mathbf{7 1 2}$ passive right ventricular leads, mainly in the apex. After a mean follow-up of $54.9 \pm 37.6$ days, dislodgement rates were $1.0 \%$ and $1.6 \%$ for atrial and ventricular active, and $3.2 \%$ for ventricular passive leads. No unexpected adverse reactions were observed during the course of the study and the electrical performances at implant and follow-up visits remained within normal ranges. Overall, most investigators $(84 \%)$ rated leads' handling as superior

${ }^{*}$ Corresponding author. 
(better or best) to what observed with other bradycardia leads. Conclusion: Different bradycardia leads showed a dislodgement rate of $1.0 \%$ and $1.6 \%$ for atrial and ventricular active leads, and $3.2 \%$ for ventricular passive leads, at 3-month follow-up. Acute safety and electrical performances were within expected ranges and very good handling performances were observed.

\section{Keywords}

\section{Bradycardia Lead, Acute Lead Performance, Lead Handling, Lead Safety, Active Fixation Lead, Passive Fixation Lead, X-Fine, Beflex}

\section{Introduction}

With a broadening of the indications for bradycardia and tachycardia management and an aging population, the number of pacemakers and cardioverter defibrillators implants is increasing. There is also a shift toward the use of active-fixation leads, which offer the potential advantages of reduced lead dislodgment, rapid implantation and easier lead extraction compared to passive-fixation leads.

The FINE registry aimed to investigate short-term behavior of different bradycardia lead models: ventricular passive- and active-fixation and atrial active-fixation pacing leads.

\section{Methods}

\subsection{Study Design}

FINE (an observational study on XFine and Beflex bradycardia leads, ClinicalTrials.gov Identifier: NCT 01168518) was an observational prospective study conducted in 95 European centers (90\% in France and 10\% in Spain) evaluating XFine and Beflex bradycardia leads.

Included patients were those implanted with an endocavitary pacing lead (atrial and/or ventricular) connected to a single, double or triple chamber pacemaker or defibrillator. The implanted lead(s) had to allow the measurement of pacing capture threshold (PCT) at $0.5 \mathrm{~ms}$, R-wave or P-wave amplitude and impedance of the lead(s). Patients were implanted with one or two of the leads under investigation. After enrolment, patients received their implant. Electrical lead performances were assessed at implant using a Program System Analyzer. A follow-up visit was scheduled one to three months after implant, to assess the device electrical performance, and report any lead dislodgment.

The study was declared to all competent authorities in France and Spain. Enrolled patients gave their informed consent and the study conduct complied with Good Clinical Practices and the Helsinki declaration.

\subsection{Study Objectives}

The primary objective was the acute dislodgment rate of the leads during the study period (up to three months following implant).

Secondary objectives included a lead handling performance questionnaire filled-in by implanters and collected immediately after implant; the assessment of electrical performances (PCT, sensing amplitude and impedance) at implant (using a PSA) and at the follow-up visit (using the device). Adverse events were recorded throughout the study duration including extra or emergency visits.

\subsection{Implanted Devices}

The Beflex ${ }^{\mathrm{TM}}$ leads (Sorin CRM SAS, Clamart, France) is a bipolar, endocardial, 6-French lead body, steroid eluting, silicone insulated lead with an extendable (up to $1.5 \mathrm{~mm}$ )/retractable active-fixation helix for permanent pacing and sensing of either the atrium or ventricle (Figure 1). The lead is designed to be used with implantable cardiac pacemakers and defibrillators. In this steroid-eluting lead, a silicone elastomer collar containing $310 \mu \mathrm{g}$ of dexamethasone sodium phosphate is located just behind the electrode tip. Upon exposure to body fluids, the steroid elutes progressively into the cardiac tissue around the electrode. These active straight leads can be used in the ventricle (RF46D model) or atrium (RF45D model). 


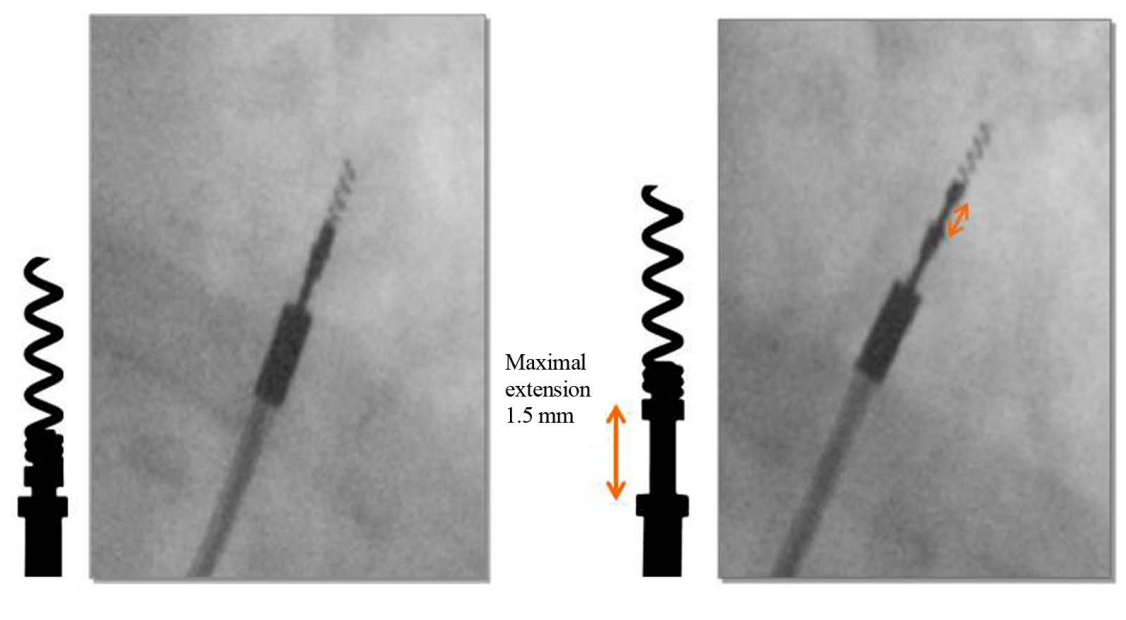

Retracted screw (on the left) and extended screw (on the right)

Figure 1. X-ray visibility-Beflex ${ }^{\mathrm{TM}}$ leads.

The XFine ${ }^{\mathrm{TM}}$ passive straight ventricular leads (Sorin CRM SAS, Clamart, France) are endocardial leads with a $4.8 \mathrm{~F}$ lead body designed to be used with implantable cardiac pacemakers. They present a steroid-eluting lead, a silicone elastomer collar containing $1 \mathrm{mg}$ of dexamethasone sodium phosphate located just behind the electrode tip. Two models were evaluated in the trial: $52 \mathrm{~cm}$ (TX 25D) and $58 \mathrm{~cm}$ (TX 26D).

The model of the pacemaker or defibrillator was left to investigators' discretion.

\subsection{Statistical Analysis}

For the primary objectives, the dislodgement rates were compared to pre-specified values, different for each type of lead, i.e., $4 \%$ [1] for active atrial leads, 1.2\% [2] for active ventricular leads and $0.7 \%$ [3] for passive ventricular leads. To reach a power of $95 \%$ for each test, and a type I error of $1.25 \%$, adjusted with a potential rate of $10 \%$ lost to follow-up or missing data, estimated sample sizes were 750 atrial active leads, 806 ventricular active leads and 1070 ventricular passive leads.

Dislodgement was recorded when either reported as an adverse event or recorded in a specific field on the CRF. When dislodgement was reported without mentioning the type of lead (atrial or ventricular) on the adverse event or follow-up form, or reported as implanted in the ventricle, but without specifying if active or passive lead in the implant CRF, the following rules were applied:

- if the patient had one implant lead, the dislodgement was attributed to the implanted lead;

- if the patient had an unknown implanted ventricular lead, the dislodgement was attributed to both ventricular passive and active types;

- if the patient had more than one implanted lead (atrial or ventricular), the dislodgement was attributed to all the implanted leads.

The analysis was performed on the Intention-to-Treat (ITT) population including all patients with at least one successfully implanted lead. Descriptive statistics were used to present and summarize the data overall and by lead type (atrial active/ventricular active/ventricular passive). All tests for the primary endpoint were one-sided, and statistical significance was considered with a risk of $2.5 \%$. No statistical test was performed when the mean value obtained exceeded the expected value.

\section{Results}

A total of 2886 leads were implanted in 2251 patients (Table 1 presents the repartition by leads' model) between June 2008 and June 2010.

\subsection{Lead Implantation}

The lead access and positions are specified in Table 1 and Table 2. Approximately $40 \%$ of the leads were implanted through the cephalic vein. 
Table 1. Lead access at implantation.

\begin{tabular}{lccc}
\hline & $\begin{array}{c}\text { Atrial active } \\
\mathbf{( N ~ = ~ 1 1 5 3 )}\end{array}$ & $\begin{array}{c}\text { Ventricular active } \\
\mathbf{( N ~ = ~ 1 0 2 1 )}\end{array}$ & $\begin{array}{c}\text { Ventricular passive } \\
\mathbf{( N = 7 1 2})\end{array}$ \\
\hline Access vein & & & \\
Subclavian vein & $690(61.6 \%)$ & $572(57.5 \%)$ & $422(60.6 \%)$ \\
Cephalic vein & $423(37.8 \%)$ & $423(42.5 \%)$ & $272(39.0 \%)$ \\
Other & $7(0.6 \%)$ & - & $3(0.4 \%)$ \\
Missing data & $33(2.9 \%)$ & $26(2.5 \%)$ & $15(2.1 \%)$ \\
Access side & & & \\
Left & $643(65.8 \%)$ & $526(58.1 \%)$ & $404(64.9 \%)$ \\
Right & $334(34.2 \%)$ & $379(41.9 \%)$ & $219(35.1 \%)$ \\
\hline
\end{tabular}

\begin{tabular}{cccc|}
\multicolumn{4}{l}{ Table 2. Lead position per model. } \\
\hline Position & $\begin{array}{c}\text { Atrial active } \\
\mathbf{( N = 1 1 5 3 )}\end{array}$ & $\begin{array}{c}\text { Ventricular } \\
\text { active (N = 1021) }\end{array}$ & $\begin{array}{c}\text { Ventricular passive } \\
\mathbf{( N = 7 1 2 )}\end{array}$ \\
\hline Atrial lateral wall & $172(15.7 \%)$ & $/$ & $/$ \\
Right auricle & $872(79.6 \%)$ & $/$ & $/$ \\
Ventricular apex & $/$ & $294(29.9 \%)$ & $677(97.7 \%)$ \\
Ventricular septum & $/$ & $673(68.5 \%)$ & $5(0.7 \%)$ \\
Other* & $52(4.7 \%)$ & $16(1.7 \%)$ & $11(1.6 \%)$ \\
Missing & $57(4.9 \%)$ & $38(3.7 \%)$ & $19(2.7 \%)$ \\
\hline
\end{tabular}

*Positioned in the wrong chamber.

General opinion on the lead handling was good. Overall most investigators rated the leads as "better" to implant compared to other models (general opinion on the lead implantation rated as "the best" or "better", according to the handling questionnaire results): $83.8 \%$ for the atrial active lead, $80.2 \%$ for the ventricular active and $90.0 \%$ for the ventricular passive leads. Detailed results on main items of the questionnaire are summarized in Figure 2.

\subsection{Primary Objective}

During a mean duration of $54.9 \pm 37.6$ days, 12 dislodgements (1.0\%) were reported with the atrial active leads (95\% confidence interval (CI): [0.5\% - 1.6\%], $\mathrm{p}<0.0001, \mathrm{n}=1153)$; 16 dislodgements (1.6\%) were reported with the right ventricular active leads (95\% CI: [0.8\% - 2.3\%], $\mathrm{n}=1021)$; 23 dislodgements (3.2\%) were reported with the right ventricular passive leads $(95 \%$ CI: [1.9\% - 4.5\%], $n=712)$. The required sample size of 1070 ventricular passive leads was not reached.

\subsection{Secondary Objectives}

Electrical performances of the leads are presented in Table 3. They remained within expected range up to the follow-up visit for the 3 models investigated.

While there was no device or procedure related death reported; six neither device-, nor procedure-related deaths $(0.3 \%)$ occurred during the study (three cardiovascular deaths, one pulmonary death and one not specified). In addition to the dislodgements reported as SAEs and already analyzed in the primary endpoint, four procedure-related SAEs were reported (three pocket hematoma and one infection) and five SAEs neither device, nor procedure related occurred, including one cardiovascular in nature.

\section{Discussion}

The FINE registry is an observational, prospective, multicenter clinical investigation on bradycardia leads aiming 
Table 3. Electrical performances of the leads at implant and at follow-up (between 1 and 3 months after implant).

\begin{tabular}{lcrcr}
\hline & Pacing Thresholds & Impedance & Sensed Amplitude \\
\hline & \multicolumn{4}{c}{ Atrial active lead, $\mathbf{n}=1153$} \\
At implant & $0.92 \pm 0.64 \mathrm{~V}$ & $612.98 \pm 169.52 \Omega$ & $3.12 \pm 1.84 \mathrm{mV}$ \\
Follow-up & $0.69 \pm 0.40 \mathrm{~V}$ & $496.55 \pm 123.29 \Omega$ & $3.18 \pm 1.53 \mathrm{mV}$ \\
& \multicolumn{4}{c}{ Ventricular active lead, $\mathbf{n}=\mathbf{1 0 2 1}$} \\
At implant & $0.64 \pm 0.34 \mathrm{~V}$ & $794.16 \pm 217.46 \Omega$ & $11.03 \pm 5.53 \mathrm{mV}$ \\
Follow-up & $0.74 \pm 0.50 \mathrm{~V}$ & $602.53 \pm 171.32 \Omega$ & $10.80 \pm 3.69 \mathrm{mV}$ \\
& \multicolumn{4}{c}{ Ventricular passive lead, $\mathbf{n}=\mathbf{7 1 2}$} \\
At implant & $0.43 \pm 0.31 \mathrm{~V}$ & $811.95 \pm 256.28 \Omega$ & $12.24 \pm 5.51 \mathrm{mV}$ \\
Follow-up & $0.66 \pm 0.58 \mathrm{~V}$ & $593.49 \pm 137.16 \Omega$ & $11.21 \pm 3.68 \mathrm{mV}$ \\
\hline
\end{tabular}

EASE OF USE WITH RECOMMENDED INTRODUCER

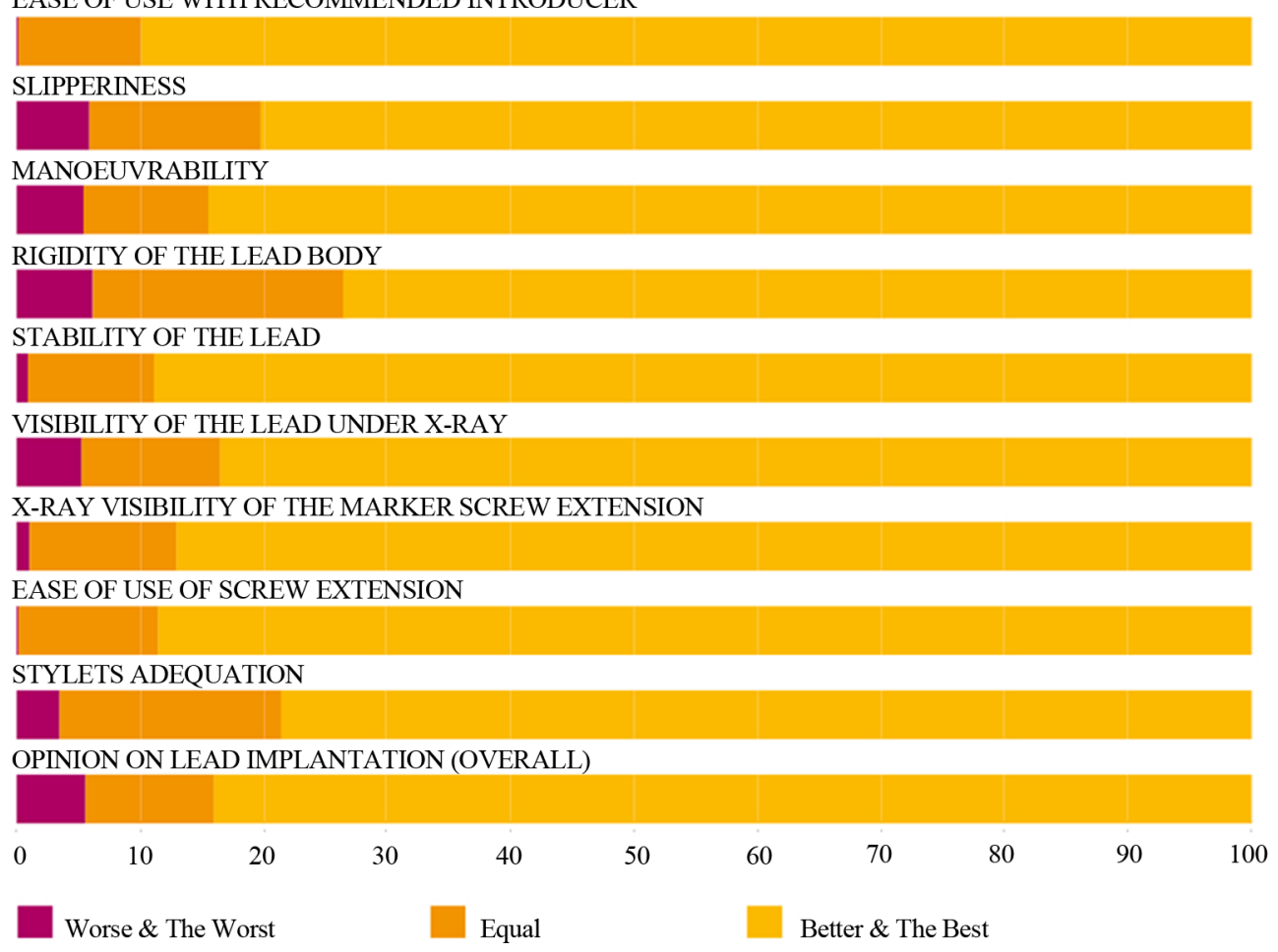

Figure 2. Results of the lead handling questionnaire collected at implantation.

to evaluate lead performances and safety in acute conditions. This post-market evaluation was conducted in 2254 patients implanted with 2886 leads in France and Spain from 2008 and 2010. Patients were followed-up for one to three months (one single follow-up visit). The dislodgement rates were respectively $1.0 \%$ for atrial, $1.6 \%$ for ventricular active and 3.2\% for ventricular passive leads. No unexpected adverse reactions were observed during the course of the study and the electrical performances at implant and follow-up visits remained within expected ranges. The handling of the leads for highly rated by the implanters.

The dislodgment rates of the Beflex atrial and ventricular leads were as expected and within the range of the ones reported in the literature: within 30 days after implant, from $0.1 \%$ to $2.6 \%$ for the atrial lead [4]-[7] and from $0.3 \%$ to $1.7 \%$ [7] [8] for the ventricular lead. The XFine passive ventricular lead presented a higher dislodgment rate than expected (3.2\%). However, it should be noted that, due to a lower inclusion rate as initially scheduled, the required sample size was not reached. Thus this section of the study did not attain a power of $95 \%$ to 
support our results.

Electrical performances were within ranges retrieved in the literature for the atrium: PCT was comparable to values observed by Kistler et al. [9], P-wave amplitude were close to those reported by Rickard [6] and Cornacchia [10], and impedance values compared to those found by Kistler [9] and Lotze et al. [11]. In the ventricle, PCT values compared with those reported by Rickard [6] and Lotze et al. [11]; sensing amplitude were aligned with Nagatomo et al. [12] and Schuchert et al. [13]. Finally, impedances were close to those reported by Schuchert [13].

The ease with which a lead can be introduced into the cephalic vein depends on several factors including anatomical considerations, tortuosity; the vein's status; cardiologist's experience and lead trackability. In the FINE registry, approximately $40 \%$ of the leads were successfully implanted through the cephalic vein. Routing the leads by the cephalic vein can be an interesting option for cardiologists as it avoids subclavian ponction with all the possible complications, such as pneumothorax, hematoma, arterial injury and damage linked to costoclavicular crush. The success of using the cephalic vein should not be interpreted solely using percentage achievement rates in a quantitative way, but should also include the qualitative evidence that shows the incontestable facility of using this route.

\section{Limitations of the Study}

By design, the registry presents several limitations: e.g. no ongoing monitoring was performed, and it led to a large amount of missing data in the different parameters collected and important indication on the study population, such as demographics were not collected. Moreover, in the cases of missing/not comprehensible data, the most conservative approach was chosen and therefore, the incidence of lead dislodgment reported might be superior to the reality. Finally, the required sample size could not be reached for the XFine lead due to a low inclusion rate.

\section{Conclusion}

The three different bradycardia leads studied in the FINE registry showed a dislodgement rate of $1.0 \%$ and $1.6 \%$ for atrial and ventricular active leads, and 3.2\% for ventricular passive leads, at 3-month follow-up. Very good handling performances were observed and acute safety and electrical parameters were within expected ranges.

\section{Acknowledgements}

The authors thank Anne Rousseau-Plasse, PhD and Frédérique Maneval, MSc, for editorial assistance.

\section{Conflict of Interest}

The FINE registry wad sponsored by SORIN CRM SAS (Clamart, France).

\section{References}

[1] Mond, H.G., Hua, W. and Wang, C.C. (1995) Atrial Pacing Leads: The Clinical Contribution of Steroid Elution. Pacing and Clinical Electrophysiology, 18, 1601-1608. http://dx.doi.org/10.1111/j.1540-8159.1995.tb06981.x

[2] Lipchenca, I.A., Bar-Lev, D.S., Luria, D.M., Gurevitz, O.T., Granit, C., Rotstein, Z., et al. (2006) Clinical Surveillance of a Tined, Bipolar, Silicone-Insulated Ventricular Pacing Lead. Journal of the Working Groups on Cardiac Pacing, Arrhythmias, and Cardiac Cellular Electrophysiology of the European Society of Cardiology, 8, 118-121. http://dx.doi.org/10.1093/europace/euj029

[3] Ellenbogen, K.A., Hellkamp, A.S., Wilkoff, B.L., Camunas, J.L., Love, J.C., Hadjis, T.A., et al. (2003) Complications Arising after Implantation of DDD Pacemakers: The MOST Experience. The American Journal of Cardiology, 92, 740-741. http://dx.doi.org/10.1016/S0002-9149(03)00844-0

[4] Daubert, C., Gold, M.R., Abraham, W.T., Ghio, S., Hassager, C., Goode, G., et al. (2009) Prevention of Disease Progression by Cardiac Resynchronization Therapy in Patients with Asymptomatic or Mildly Symptomatic Left Ventricular Dysfunction: Insights from the European Cohort of the REVERSE (Resynchronization Reverses Remodeling in Systolic Left Ventricular Dysfunction) Trial. Journal of the American College of Cardiology, 54, 1837-1846. http://dx.doi.org/10.1016/j.jacc.2009.08.011

[5] Stefano, B., Pietro, R.R., Maurizio, G., Maurizio, L., Renato, M., Maurizio, L., et al. (2011) Defibrillation Testing 
during Implantable Cardioverter-Defibrillator Implantation in Italian Current Practice: The Assessment of Long-Term Induction Clinical Value (ALIVE) Project. American Heart Journal, 162, 390-397. http://dx.doi.org/10.1016/j.ahj.2011.04.008

[6] Rickard, J., Taborsky, M., Bello, D., Johnson, W.B., Ramza, B., Chang, Y., et al. (2014) Short- and Long-Term Electrical Performance of the 5086MRI Pacing Lead. Heart Rhythm, 11, 222-229. http://dx.doi.org/10.1016/j.hrthm.2013.10.056

[7] Ghani, A., Delnoy, P.P., Ramdat Misier, A.R., Smit, J.J., Adiyaman, A., Ottervanger, J.P., et al. (2014) Incidence of Lead Dislodgement, Malfunction and Perforation during the First Year Following Device Implantation. Netherlands Heart Journal, 22, 286-291. http://dx.doi.org/10.1007/s12471-014-0556-6

[8] Zoppo, F., Zerbo, F., Brandolino, G., Bacchiega, E., Lupo, A. and Bertaglia, E. (2011) Straight Screw-In Atrial Leads "J-Post Shaped" in Right Appendage versus J-Shaped Systems for Permanent Atrial Pacing: A Safety Comparison. Pacing and Clinical Electrophysiology, 34, 325-330. http://dx.doi.org/10.1111/j.1540-8159.2010.02986.X

[9] Kistler, P.M., Liew, G. and Mond, H.G. (2006) Long-Term Performance of Active-Fixation Pacing Leads: A Prospective Study. Pacing and Clinical Electrophysiology, 29, 226-230. http://dx.doi.org/10.1111/j.1540-8159.2006.00327.x

[10] Cornacchia, D., Fabbri, M., Puglisi, A., Moracchini, P., Bernasconi, M., Nastasi, M., et al. (2000) Latest Generation of Unipolar and Bipolar Steroid Eluting Leads: Long-Term Comparison of Electrical Performance in Atrium and Ventricles. Journal of the Working Groups on Cardiac Pacing, Arrhythmias, and Cardiac Cellular Electrophysiology of the European Society of Cardiology, 2, 240-244. http://dx.doi.org/10.1053/eupc.2000.0103

[11] Lotze, U., Fischer, S., Hofs, T., Liebetrau, J., Fettin, W., Scheiner, J., et al. (2009) Electrical Performance and Automatic Capture Characteristics of a 3.5-mm(2) Passive Fixation Lead during 1-Year Follow-Up. Pacing and Clinical Electrophysiology, 32, 1050-1055. http://dx.doi.org/10.1111/j.1540-8159.2009.02437.x

[12] Nagatomo, T., Abe, H., Numata, T., Tamura, K., Kikuchi, K., Oginosawa, Y., et al. (2000) Clinical Surveillance of a Thin Bipolar Pacing Lead. Japanese Circulation Journal, 64, 933-936. http://dx.doi.org/10.1253/jcj.64.933

[13] Schuchert, A., Muto, C., Maounis, T., Frank, R., Boulogne, E., Polauck, A., et al. (2013) Lead Complications, Device Infections, and Clinical Outcomes in the First Year after Implantation of Cardiac Resynchronization Therapy-Defibrillator and Cardiac Resynchronization Therapy-Pacemaker. Journal of the Working Groups on Cardiac Pacing, Arrhythmias, and Cardiac Cellular Electrophysiology of the European Society of Cardiology, 15, 71-76.

\section{List of Abbreviations}

\begin{tabular}{ll}
\hline AE & Adverse Event \\
CRF & Case Report Form \\
ECG & Electrocardiogram \\
PHD & Pre Hospital Discharge \\
PSA & Program System Analyzer \\
ITT & Intention-To-Treat \\
SAP & Statistical Analysis Plan \\
SD & Standard Deviation \\
M1 & Month 1 \\
M3 & Month 3 \\
\hline
\end{tabular}

